\title{
La relación bilateral entre Chile y el Perú: La propuesta del fallo de la Corte Internacional de Justicia como punto de inflexión histórico y su posterior fracaso
}

\author{
The bilateral relationship between Chile and Peru: the proposal of the \\ International Court of Justice judgment as a historical turning point and \\ its posterior failure
}

\author{
Rocío Arenas Riveros* \\ Consejo Académico Consultivo de Estudios e Investigación Militar \\ Pablo Rivas Pardo** \\ University of Groningen, Reino de los Países Bajos
}

Recibido: 17 de julio de 2015. Aprobado: 20 de mayo de 2017

\begin{abstract}
Resumen
Entre los meses de enero de 2008 y enero de 2014 se vivió entre el Perú y Chile un contencioso en la Corte Internacional de Justicia. Si bien el fallo no dio todo lo solicitado al Perú, este país sí logró la incorporación de un nuevo espacio marítimo en desmedro de Chile. En este lapso de seis años surgió la propuesta de que el fallo de la Corte fuera un punto de inflexión que cambiaría una relación que históricamente ha sido de confrontación e inconstancia integrista a una, más bien, de base estructural cooperativa. La hipótesis no se aprobó, puesto que ocurrido el fallo inmediatamente se levantó un

Cientista Política por la Universidad Diego Portales; Diplomado en Relaciones Internacionales, Universidad de Chile; Diplomado en Seguridad Multidimensional, Academia Nacional de Estudios Políticos y Estratégicos. Actualmente se desempeña como asesora en el Consejo Académico Consultivo de Estudios e Investigación Militar del Ejército de Chile. Correo electrónico: rocio.arenas@gmail.com

** Cientista Político y Licenciado en Ciencia Política de la Universidad Diego Portales. Diplomado en Operaciones de Paz y Seguridad Internacional, Diplomado Seguridad y Defensa, ambos en la ANEPE; Diplomado Corresponsal de Defensa, Academia de Guerra del Ejército; y Diplomado La relación chileno-peruana: puntos de encuentro, Universidad de Chile. Actualmente es alumno del Master of Art International Relations and International Organization, University of Groningen, Reino de los Países Bajos. Correo electrónico: p.rivas.pardo@ gmail.com
\end{abstract}


nuevo conflicto, el denominado "triángulo terrestre", que eliminó la propuesta del punto de inflexión. En virtud de lo anterior, este artículo explica por qué no se logró cumplir con el punto de inflexión, en razón del denominado triángulo terrestre. El análisis del rechazo del punto de inflexión incluye los principales eventos de esta relación bilateral, explicando así, por qué un triángulo de 3,7 hectáreas tuvo un valor cualitativo mayor que cambiar una relación bilateral caracterizada por su inconstancia.

Palabras clave: Relación Chile-Perú, punto de inflexión, triángulo terrestre.

\begin{abstract}
During January 2008 and January 2014, Peru and Chile lived a dispute in the International Court of Justice. Although the ruling did not give everything Peru request, this country achieved incorporated a new maritime space at the expense of Chile. In this six years, arose the hypothesis that the Court's decision would be a turning point that would change a confrontational and inconstancy relationship to new bilateral relationship with cooperative structure. The hypothesis was not approved, because immediately a new conflict arose, the called "terrestrial triangle" which eliminated the thesis of the inflection point. In view of the above, this article explains why it was not possible to comply with the turning point, because of the terrestrial triangle. The analysis of the turning point includes the main events of this bilateral relationship, it explaining in this way, why a triangle of 3,7 hectares has a qualitative value bigger than to change a bilateral relationship characterized by its inconstancy.
\end{abstract}

Keywords: Chile-Peru relationship, turning point, land triangle.

\title{
Introducción
}

El Perú interpuso ante la Corte Internacional de Justicia (CIJ) en enero de 2008, una demanda contra el Estado de Chile. Tal demanda, en síntesis, solicitó que la CIJ definiera el límite marítimo entre ambos países; especificara el origen de tal demarcación; que realizara la proyección de una línea equidistante; y que tal proyección abarcara aguas internacionales como parte de sus aguas nacionales (CIJ, 2008). En medio de este contexto 
y previo al fallo de la CIJ, se desarrolló y difundió, durante el gobierno del Presidente de Chile, Sebastián Piñera (2010-2014) y del Perú, Alan García (2006-2011) y luego Ollanta Humala (2011-2016), la hipótesis de aprovechar la coyuntura de la demanda como un momento oportuno para generar un cambio en la relación bilateral respecto a la conformación territorial, es decir "un punto de inflexión". Lo anterior fue una iniciativa que se adoptó a nivel presidencial, entre cancillerías y el plano académico.

Finalmente, la Corte dio a conocer su fallo el 27 de enero de 2014 (ver anexo I). Si bien el origen del límite no fue el propuesto por el Perú, ya que fue un punto ubicado al norte del solicitado, y la línea equidistante comienza 80 millas marítimas después de la línea paralela que va desde el origen, el Estado peruano incorporó espacio marítimo a su soberanía territorial, económica y jurisdiccional, que el Estado de Chile consideraba como propio (CIJ, 2014).

Sin embargo, tras el fallo, y a semanas de ser conocido el resultado de la demanda, surgió una nueva situación de tensión, el "denominado triángulo terrestre" (ver anexo II), que ambos países consideraron como propio, no siendo efectiva la propuesta de que el fallo sería el punto de inflexión en las relaciones bilaterales.

Luego de dicho desencuentro, la posibilidad de aprovechar la coyuntura de la demanda como un punto de inflexión que concluyera con el ciclo de disputas a lo largo de la historia de la relación bilateral no funcionó, en el inmediato ni en el corto plazo.

Cabe distinguir que se usa la expresión "denominado triángulo terrestre" dado que el espacio territorial no corresponde geométricamente a un triángulo y que además, ambos países lo consideran como propio, por lo cual, la existencia o no de un triángulo terrestre pendiente corresponde a que ambos Estados así lo digan.

El artículo utiliza una metodología cualitativa de investigación, y el método de estudio de caso, para examinar una relación específica en un período de tiempo determinado entre dos actores: la relación bilateral entre Chile y el Perú. El objetivo general de este artículo es explicar por qué el fallo de la CIJ no fue un punto de inflexión en la relación bilateral que se esperaba, y consecuentemente, identificar el motivo del fracaso del "triángulo terrestre". Los objetivos específicos son 1) Especificar el concepto punto de inflexión, 2) Analizar la propuesta del triángulo terrestre como punto de inflexión, 3) Examinar los ciclos de integración y diputas de la relación bilateral con el objeto de comprender la incidencia del pasado en la situación actual. Estos objetivos buscan responder a la 
siguiente pregunta: ¿Qué explica que el triángulo terrestre no permitiera la posibilidad de punto de inflexión histórico en la relación bilateral?

\section{Punto de inflexión: definición, propuestas políticas y académicas}

El concepto punto de inflexión posee diferentes significados según el contexto en que se utiliza, por ejemplo, en geometría inflexión significa "el punto de una curva en que cambia de sentido su curvatura" (RAE, 2014). En el lenguaje anglosajón, punto de inflexión se denomina turning point y se define como "el momento en que una situación comienza a cambiar en un sentido importante" (Cambridge Dictionary, 2014) en otras palabras "punto en el que se produce un cambio significativo" (Merriam Dictionary, 2014) o "verdaderos cambios que han transformado la manera en que son percibidas las sociedades humanas" (Guillén y Ontveros, 2012: 9), es decir, en el plano de las ciencias sociales, esto se considera como un cambio importante en la dirección de un fenómeno social. Desde la historia, Roger Launius señala que los puntos de inflexión "son un evento o varios de ellos que son capaces de cambiar la historia; es un hecho particular y no necesariamente debe tener la cualidad de dramático" (Launius, 2007: 19). En este artículo se utiliza el concepto como un evento que demarca un cambio significativo que circunscribe un antes y después en la historia de la relación bilateral entre Chile y el Perú.

En el caso citado, durante el gobierno del presidente Piñera por Chile y los presidentes García (2006-2011) y Humala (2011-2016) por el Perú, se propuso transformar el problema de la demanda en una oportunidad o "punto de inflexión" para lograr un cambio en la relación que a lo largo del tiempo se ha caracterizado por ser intermitente, teniendo un carácter tenso y de confrontación.

Marcos Robledo, entonces académico de la Universidad Diego Portales y actual Subsecretario de Defensa de Chile, fue explícito al escribir sobre esta idea, señalando que:

"La sentencia de la Corte Internacional de Justicia podría, e idealmente debería, convertirse en un punto de inflexión histórico que marque una nueva etapa, en que la conformación territorial de los Estados peruano y chileno es aceptada por ambas partes" (Robledo, 2012: 30).

116 Si Somos Americanos. Revista de Estudios Transfronterizos 
Si bien la cita apunta a que se puede lograr un cambio, se infiere que no existiría o de plano no existe entre ambos países, una opinión compartida sobre la conformación territorial. Lo anterior fue comprobado tras el fallo de la CIJ, ya que el denominado "triángulo terrestre" no dio espacio al punto de inflexión esperado.

Relacionado con lo anterior, en noviembre de 2012, durante la VI Cumbre de UNASUR -a tres días antes de la fase oral del juicio- ambos Presidentes hicieron declaraciones con la intención de mejorar la relación bilateral, por su parte el Presidente Humala indicó:

"Hoy día estamos trabajando por fortalecer la integración, la relación bilateral entre Chile y el Perú, fortalecer el dinamismo de nuestras economías y en general, hacer más fructífera esta relación bilateral (...) Señalamos la importancia de trabajar una agenda después de La Haya" (El Mercurio: 2012).

El Presidente Piñera respondió:

"La agenda del pasado a veces nos ha dividido, pero la agenda del futuro definitivamente nos une, hemos conversado con el Presidente Humala cómo seguir fortaleciendo los lazos de unidad, amistad y colaboración entre Perú y Chile" (El Mercurio: 2012).

A nivel de Cancilleres, en enero de 2013, estos también manifestaron su voluntad de conciliar un futuro auspicio en las relaciones bilaterales:

"Los Ministros reafirmaron, en ese sentido, la voluntad de sus gobiernos de trabajar coordinadamente en la generación de las condiciones más propicias para consolidar y proyectar en el mediano y largo plazo una relación bilateral de estabilidad, confianza y cooperación" (Perú, Ministerio de Relaciones Exteriores: 2013).

Durante los años 2010 y 2013, se realizaron, en ambos países, considerables charlas, mesas de trabajo y seminarios sobre la relación de Chile y Perú, orientado a las posibles sentencias de la Corte y las repercusiones a futuro para las dos naciones. De esto se destacan los encuentros binacionales de distintas personalidades de Chile y el Perú, tanto en Lima como en Santiago y la invitación de Académicos peruanos y ex Ministros. En esto, se reconoce en particular el aporte a la materia que realizó el Instituto de Ciencias Sociales de la Universidad Diego Portales (ICSO UDP), el Instituto de Estudios Internacionales de la Universidad de Chile (IEI), el Instituto de Estudios Internacionales 
de la Pontificia Universidad Católica del Perú (IDEI PUCP), la Fundación Chile 21 y la Fundación Konrad Adenauer (KAS).

Dentro de las anteriores se encuentra la charla realizada en el ICSO UDP, donde expusieron los ex Cancilleres Rafael Roncagliolo del Perú (2011-2013) y Gabriel Valdés por Chile (1999-2000). Ambos coincidieron en ver el fallo de la CIJ como oportunidad. Aun así se plantearon incertidumbres, la del Perú consistía en un eventual armamentismo chileno, y por parte de Chile, el que esta no fuese la última disputa territorial por parte del Perú (ICSO UDP, 2013).

Reforzando aquello, el historiador peruano Daniel Chasqui aseveró frente a la idea de un cambio en la relación:

“¡Claro que se puede lograr (el punto de inflexión) y ya se está logrando! Si ambos países acatamos el fallo y lo ejecutamos con prontitud y humildad, las buenas historias nos buscarán a nosotros. En cambio, si comenzamos a ponerle "peros" al fallo y comenzamos a dilatar la ejecución, la desconfianza se va a acrecentar" (Publimetro Perú, 2014).

Hay claridad en que, desde el plano político como académico, hubo una real intención de que el punto de inflexión histórico, que invitaba a cambiar la relación bilateral en el contexto de la demanda del Perú contra Chile, fuese concebido, sin embargo, en razón del denominado "triángulo terrestre" no fue posible en el inmediato o corto plazo.

\section{El triángulo terrestre como impedimento para cambiar la relación bilateral}

Inmediatamente dado el resultado de la CIJ, en enero de 2014, surgió una nueva controversia: el denominado triángulo terrestre (ver anexo II). Fue así que toda hipótesis de concluir los temas pendientes en materia de conformación territorial quedó desestimada. En el contexto de la VIII Cumbre de la Alianza del Pacífico, realizada en febrero de 2014 en Cartagena de Indias, los Presidentes Humala y Piñera hablaron sobre el triángulo. Tras el encuentro bilateral Humala señaló «hemos conversado, cada uno tiene su posición, pero llevando la voz del Estado peruano le hemos ratificado con firmeza al presidente Piñera la soberanía de Perú sobre el denominado triángulo terrestre» (El 
Mercurio, 2014), a lo que el gobierno de Chile por medio de su Presidente contestó con nota de protesta y de manera pública:

"Le hemos expresado a (Ollanta) Humala que la posición peruana no está de acuerdo al derecho, ni siquiera a lo que ha sido la jurisprudencia durante más de 60 años respecto de ese triángulo terrestre (...) Estoy convencido de que los argumentos chilenos en esta materia son muy sólidos" (La Tercera, 2014).

Meses después, el 19 de agosto de 2014, el Presidente Humala, en acto público, en el Salón Cáceres, mostró una nueva cartografía de los límites peruanos. En esta, incluyó el denominado triángulo terrestre como parte de la soberanía peruana e indicó:

"Esta carta recoge la sentencia de La Haya del 27 de enero de este año y los trabajos que ha venido realizando una Comisión Mixta peruano-chilena para determinar las coordenadas del límite marítimo (...) esta Carta señala claramente que el inicio de la frontera entre Perú y Chile es el Punto Concordia y no hay otro" (Perú, Presidencia, 2014).

Lo anterior deja explícito la falta de intención de promover una buena relación o dejar lugar a que se produjera el punto de inflexión. La respuesta del Estado de Chile, otorgada por el Canciller Heraldo Muñoz, señaló que "el triángulo terrestre es incuestionablemente chileno" (La Tercera, 28 de agosto de 2014), a propósito de una marcha convocada por un grupo nacionalista peruano para ir desde Tacna al triángulo terrestre, iniciativa que finalmente no llegó al destino propuesto.

En este contexto, vemos que la propuesta de un punto de inflexión, como instancia para concluir la conformación territorial de ambos Estados, no se logró, en primer lugar, en razón de un triángulo terrestre -que mide 3,7 hectáreas- y en segundo lugar, a que ninguno de los gobiernos está dispuesto a priorizar unas buenas relaciones bilaterales por sobre la oportunidad de reclamar un espacio territorial como propio, puesto que es parte de su idiosincrasia.

Respecto a la pregunta de investigación, este artículo tiene la hipótesis de que la propuesta del triángulo terrestre como punto de inflexión, que concluiría el ciclo de disputas a lo largo de la historia de la relación bilateral respecto a la conformación territorial, no fue viable de acuerdo a la incidencia del pasado en la situación actual. 


\section{Ciclos de integración y diputas de la relación bilateral entre Chile y el Perú}

El artículo considera como un factor fundamental estudiar la incidencia del pasado en la situación actual, puesto que permite comprender que no es ajeno a la historia entre Chile y el Perú, el que no se terminen los asuntos pendientes en el ámbito territorial. Por tanto, que surja un nuevo tema en la agenda y que no se haya concretado la propuesta del punto de inflexión, no es una novedad, aún más, considerando que hubo académicos que advirtieron que dicho triángulo sería un nuevo punto en cuestión.

Se considera importante repasar dos contextos históricos. El primero es el origen de esta relación, desde la época colonial hasta el siglo XIX, la cual muestra que desde sus cimientos la relación bilateral ha estado marcada por desentendimientos. El segundo, consiste en los principales hitos del siglo XX, que denota la dificultad de la aplicación e implementación de los tratados y de los procesos de integración.

\section{El origen de la relación: colonia, independencia y las guerras}

El inicio de la relación política entre ambos países data del Siglo XVI, cuando los españoles instalaron, en el nuevo continente, las colonias. En esta etapa el Perú se constituyó en un Virreinato, principal unidad administrativa en las nuevas tierras, destacándose por ser un gran proveedor de oro para España. De manera distinta, Chile fue una gobernación que funcionó bajo la lógica militar, a causa de la lucha contra los mapuches, constituyéndose así en un reducto que demandaba recursos al Rey.

En aquella época, el Virreinato no sólo tenía una situación de desarrollo superior sobre la Gobernación de Chile, este último, además, se encontraba dependiente a las decisiones políticas y económicas que se le dictaran. Como señaló Alberto Wagner de Reyna: "La Capitanía General o reino de Chile dependió de Lima hasta 1798, la élite de sus hombres se educó en Lima, y separado de la Argentina por la cordillera, su vecino más efectivo era el Perú" (Winter, 2013: 7). A juicio de varios autores, tal situación administrativa (de dependencia) generó la instauración de una visión negativa entre ambas sociedades, la que habría sido heredada al conformarse y confrontarse las repúblicas. Esto se ejemplifica en la reseña que realizó Paz Milet al citar una entrevista del ex Canciller peruano, García Belaúnde: 
"Podría sostenerse que el establecimiento de las primeras instituciones civiles en el Perú tuvo como antecedente una guerra civil entre los pizarristas de Lima y Cuzco y "los de Chile", como eran llamados los almagristas, que derivó en el asesinato de Pizarro" (Milet, 2005: 7).

Con esta cita, se demuestra que junto con existir un origen competitivo y de subordinación entre el Virreinato del Perú y la Gobernación de Chile, existió tempranamente un enfrentamiento armado entre ambas comunidades, asunto que culturalmente es heredado una vez finalizada la época colonial. El Embajador peruano, Juan Miguel Bákula, refuerza esta idea en su libro "Perú en el Reino Ajeno" como se puede apreciar en la siguiente cita:

"La "herencia colonial", como consecuencias de la concepción política reinante, de las costumbres administrativas y de un ejercicio de la autoridad destinado a sostener una relación radial (...) Fue característico que la vinculación entre las dependencias coloniales estuviera marcada por el signo de la subordinación y, en otras circunstancias, por la prohibición" (Bákula, 2006: 97).

El caso de los almagristas y del monopolio económico que duró casi dos siglos y medios, caló en la categorización y rivalidad de las sociedades, desde un plano social y económico. Respecto a lo primero, existía una mirada despectiva hacia los "mapochinos" es decir, los residentes de Santiago, por parte de la sociedad limeña, como sinónimo de incivilizados; y en lo económico, el control que ejercía el Virreinato sobre la gobernación era total, inclusive abarcaba hasta el Puerto de Buenos Aires. Entonces, el cambio de Gobernación a Capitanía, para la élite peruana se interpretó como una manifestación de rivalidad, puesto que para los entonces "chilenos" este paso implicó tener una mejor y mayor autonomía, junto con una relación directa con la Corona y sus instituciones, que pasaron a ser representantes para el continente y ya no con el Virrey.

Cerrada la colonia, se observa claramente que esta no legó una relación cooperativa para ambos países al iniciarse la emancipación americana, al contrario, las elites tenían una mirada de rivalidad.

Posteriormente, la independencia de América del Sur, dada entre los años 1809 y 1826, tampoco heredó una estructura de buena vecindad a pesar de la ayuda prestada por Chile a la independencia del Perú, tal y como lo señala el decreto "Tratado de Auxilio al Perú" de 1823 por el cual se ayuda con medios militares a la guerra. De ese documento se destaca la inspiración política del momento: 
"Queriendo manifestar la persuasión invariable en que se halla este gobierno y todo chileno, de que la Guerra del Perú es la Guerra de Chile, no solo por la consideración de su seguridad, sino porque la gloria que ha resultado a Chile de haber sido el primero que la emprendió, se mancharía si floja o caprichosamente abandonemos el campo" (Chile. Biblioteca del Congreso Nacional, 1823: 1).

Es común suponer que la participación de Chile para lograr la expulsión de los españoles en el último reducto castellano llevó a una valorización entre las partes, al mirarse como gobiernos amigos, pero aquello es totalmente erróneo. Si se observa bien, el proceso de independencia del Perú es exportado desde el sur y del norte, no es un proceso innato a sus líderes políticos. Vale observar que son San Martín, bajo el financiamiento de O'Higgins, y Simón Bolívar con las tropas grancolombianas, los que se encuentran en Guayaquil para decidir el futuro del Virreinato. Por lo cual, el paso de Virreinato a República no deja ser una sensación errada de común ideario de vecindad.

Al decenio siguiente, las nacientes repúblicas se encuentran en la "Guerra contra la Confederación Perú-Boliviana”, dada entre 1836 y 1839. Tal episodio es visto, por la historiografía chilena más tradicional, como un punto positivo. En palabras simples, se expresa que Chile se alió con los peruanos restauradores y logró reintegrar al Perú republicano en contra del enemigo común: la Bolivia del Mariscal Santa Cruz, liberándolos por segunda vez, ya que antes había sido de España. Una segunda mirada que se presenta en parte de la historiografía peruana, es mirar la Confederación como un proyecto de integración, al cual Chile, y en especial, Diego Portales, de manera preventiva atacó con peruanos que se oponían a otros peruanos. En cita de Percy Cayo Córdova:

\footnotetext{
"Cierto es también que aun en la historiografía peruana y boliviana hay voces que proclaman su desacuerdo o antagonismo, su cuestionamiento o inconformidad con el gran proyecto confederacional (...) Más creemos que ya es tiempo de apuntar a una reflexión más certera y comprensiva del Gran Mariscal Andrés de Santa Cruz. Loas, alabanzas y encarecimientos tantas veces presentes en estas ocasiones no deben postergar la reflexión serena sobre lo que quiso realizar" (Cayo, 2010: 194).
}

La interpretación de este autor apunta a ver a Santa Cruz como un hombre que buscó la integración, como consecuencia natural de un origen común entre ambos países.

Entrando en las fases de esta guerra, las campañas contra la Confederación fueron dos. La primera, liderada por Blanco Encalada que fracasó, debiendo capitular frente a Andrés de Santa Cruz, con la firma del "Tratado de Parcaupata". Si bien el tratado obligaba a que

\section{Si Somos Americanos. Revista de Estudios Transfronterizos}


las tropas chilenas se retirasen, poniendo así fin al conflicto, el gobierno chileno hizo una nueva campaña liderada por Manuel Bulnes, que llegó al triunfo en la Batalla de Yungay. Entonces, al no haber respetado la firma de Parcaupata, se levantó la tesis de algunos historiógrafos peruanos de que "los chilenos no respetan los tratados cuando estos no les convienen" (Zapata, 2012) idea reforzada -años más tarde- al no realizarse el arbitraje por Tacna y Arica al cumplirse los 10 años del Tratado de Ancón, detallado más adelante.

En años posteriores, el Perú vivió serios problemas con sus vecinos del norte, teniendo entre medio un conflicto con Ecuador en 1851, el que venía arrastrándose desde los años 20 contra la Gran Colombia, por lo que la preocupación principal del Perú era tener seguridad y buenas relaciones con su frontera noreste, Chile no era un problema. Ya en la década de los 60 tanto Bolivia, Chile y Ecuador fueron a la ayuda del Perú durante la Guerra contra España entre 1865 y 1866. Sin embargo tal momento solidario no llevó a institucionalizar una buena vecindad, ya que por esa misma fecha comenzó la competencia económica entre los países por el salitre, teniendo como antesala la disputa del guano. El ejemplo de la competencia fue la contención entre los puertos del Callao y de Valparaíso con miras para consolidarse como el principal puerto del Pacífico Sur y de principal plataforma exportadora de salitre. En esa misma década, se firmó entre Chile y Bolivia el Tratado de 1866, el cual fijó entre las partes como límite internacional el paralelo $24^{\circ}$ de Latitud Sur implicando para Chile y el Perú no ser países vecinos (Bolivia. Dirección Estrategia de Reivindicación Marítima-DIREMAR, 2014).

A finales de la década siguiente, en 1879, comenzó la Guerra del Pacífico con la ocupación de Antofagasta, por lo cual las hostilidades fueron primeramente entre Chile y Bolivia. Pero a causa del Tratado Secreto para una asistencia militar (Pardo por el Perú y Frías por Bolivia) entró el Perú, extendiéndose la guerra hasta la Sierra peruana. El fin de las hostilidades llegó con el Tratado de Ancón de 1883, pero no así el conflicto en un plano diplomático, al contrario, este se extendió hasta 1929. Tal extensión y dilatación de una solución definitiva implicó que el problema no sea únicamente la manera en que se gana la guerra y los tributos de ella, sino también, la manera como se construyó la paz y la cultura que ella generó. En detalle, el 20 de octubre de 1883 se firma el Tratado de Paz de Ancón, el que fijó una tregua entre las partes, la provincia de Tarapacá pasó a Chile, y se dejó pendiente la situación de Tacna y Arica:

"Dicho tratado en su artículo 3 establecía que las provincias de Tacna y Arica continuarían en posesión de Chile y sujetas a la legislación y autoridades de ese país por el término de 10 años y, una vez expirado el plazo, un plebiscito (votación popular), celebrado en estas dos provincias, decidiría si las mismas quedaban definitivamente 
bajo el dominio y la soberanía de Chile, o si continuaban siendo parte del territorio peruano. Dicho plebiscito nunca llegó a realizarse y las provincias de Tacna y Arica continuaron bajo soberanía chilena, pese a que la ocupación debió terminar en 1894» (Sandoval, 2011, web).

La Cancillería peruana en su página de Internet señala sobre la Guerra del Pacífico:

"Las relaciones diplomáticas entre el Perú y Chile se establecieron inmediatamente después de la independencia. La Guerra del Pacífico motivó la interrupción de las mismas hasta la firma del Tratado de Ancón de 1883. Una nueva interrupción se produjo entre los años 1910 y 1928 como resultado de las diferencias sobre la ejecución del artículo 3 de dicho Tratado, referido a la realización de un plebiscito para definir la soberanía de las provincias cautivas de Tacna y Arica" (Perú. Ministerio de Relaciones Exteriores, 2010).

Bákula hace una reflexión muy interesante sobre los momentos en que el Perú tuvo fuertes cambios en su vida como unidad territorial y en dos de ellos Chile es parte integrante:

"Nunca el "Perú" volvió a ser el mismo en el tiempo siguiente, cuanto por los cruentos sucesos ocurridos. $\mathrm{Y}$, al igual, porque en esos tres momentos las causas determinantes fueron exógenas, o sea que vinieron de fuera. Así fue en la conquista y subsiguiente colonización; en la revolución de la independencia, al imponerse un nuevo régimen; y en la guerra del Pacífico, al invadirse y cercenarse su territorio” (Bákula, 2006: 156).

El legado negativo de la guerra se expresa en la cultura, siendo este el mayor escollo a destrabar. El Perú consagra una identidad común y nacional por medio de sus héroes, implicando una construcción de identidad en oposición de Chile y de los chilenos. El territorio cercenado, como señala Bákula, es un cambio del cual Chile es protagonista y el antagonista de un país, el cual mantiene unas provincias prisioneras, como lo señala el Escudo del Ejército del Perú, que tiene dos hojas de su rama de laurel dobladas en señal de “dos lugares cautivos" (Perú. Ejército, 2014) interpretándose como Arica y Tarapacá.

La relevancia de la Guerra del Pacífico o Guerra contra Chile, y que no entrega la Independencia ni otras guerras para el Perú, es la construcción de héroes propios que pasan a ser los primeros próceres de su vida como República, encarnados en el Almirante Miguel Grau y el General Francisco Bolognesi, ambos Héroes de la defensa del Perú. 
Sin embargo, y como se señaló antes, el tema no quedaba allí, el cómo se construyó la paz también es un problema, y quizás, aún mayor. Ernesto Yepes (1999), destacado historiador peruano, en su libro "Un plebiscito Imposible. Tacna y Arica 1925-1926" describe lo vivido por los árbitros estadounidenses, los cuales tenían como misión solucionar la situación vivida entre las partes. Ellos en sus memorias transmiten como traba para efectuar el plebiscito en los años 20 la actitud hostil de Chile para realizar un plebiscito legítimo y sin vicios.

A modo de síntesis, entre 1879 y 1929 transcurrieron 50 años, en que hubo una Guerra de cinco años, no se hizo el plebiscito, se rompieron las relaciones y se solucionó la soberanía de Tacna y Arica de manera transaccional.

La académica Paz Milet, a su vez, señala que la relación bilateral ha sido fluctuante debido a que ha estado:

"Afectada permanentemente por la herencia del pasado y sus consecuencias más visibles - pérdida territorial, invasión chilena y consecuente obtención de trofeos de guerra- que aún son un factor determinante en el avance y desarrollo de los vínculos entre Chile y Perú" (Milet, 2004: 228).

Complementando la idea anterior, esta guerra es considerada como una de las más relevantes en la historia de la región junto con la de Triple Alianza y la del Chaco en los siglos XIX y XX respectivamente. Fernando Cavieres, historiógrafo chileno, subraya que la vida económica, post Guerra del Pacífico, se restableció rápidamente, entre Chile, Perú y Bolivia, pero no fue así en la política y quedó plasmada en la historia:

"Una política de desconfianza que se fue traspasando hacia todos los sectores de la sociedad, especialmente cuando, además, los procesos históricos de los países se fueron desarrollando con ritmos y profundidades diferentes" (Cavieres, 2006: 22).

Rodríguez Elizondo indica que el orgullo vencedor chileno no ayudó a mejorar la relación bilateral, e incluso:

"En contrapunto con el rencor peruano, amarró el desarrollo futuro de ambos países a una íntima enemistad, que se expresaría, para unos, en la obligación de conservar lo ganado y, para otros, en la necesidad de recuperar lo perdido. Ese amarre impediría 
asomarse a las posibilidades de una cooperación que los potenciara a ambos conjuntamente" (en Milet, 2004: 229).

Cerrando esta sección y explicando por qué se puede afirmar la existencia de un "vicio de origen" en la relación Chile-Perú, vale recordar la frase "el Estado hace la guerra y la guerra hace al Estado" acuñada a Charles Tilly. La primera parte de ella indica lo lógico, que son los Estados los que declaran, hacen y deciden una guerra, la segunda parte, ahonda en el problema antes mencionado, que la guerra haga al Estado implica que esta diseña una identidad común.

Bajo la lógica recién explicitada, para Chile fue Yungay la que creó al roto chileno y la identidad chilena, para el Perú fue la capitulación de Ancón de 1883 y el Tratado de 1929 lo que creó una cruzada e identidad nacional, siendo esto el vicio de origen.

Vemos que ambos países no tuvieron una integración bilateral institucionalizada antes de la guerra, por lo cual, la conflagración copó un espacio vacío entre ambos países, sumada la herencia del periodo colonial.

\section{Principales hitos del Siglo XX desde el Tratado de 1929}

La relación bilateral entre Chile y el Perú, en este lapso, se puede distinguir en tres períodos. Se considera al primero, desde 1929 a la década del 1950 con la firma del Tratado de Lima. Esta fase se destacó por significantes hitos en el ámbito territorial y marítimo que permiten describir este período como uno de importantes acuerdos y de buenas relaciones vecinales, que mirado en retrospectiva pudo haber sido una oportunidad para haber desarrollado un punto inflexión, tras la solución de la soberanía de Tacna y Arica. El segundo es a partir de la década de 1960, con el surgimiento de los regímenes militares, primero en Perú en 1968 y luego en Chile en 1973, donde las relaciones fueron de alta tensión y crisis político-militar, hasta el retorno a la democracia en 1990. El tercero se desarrolla durante la década de 1990, período en que se pone en aplicación el Tratado de 1929, y que pudo haber sido otro momento oportuno para lograr el punto de inflexión en la relación, pero tampoco fue el caso.

El primer período se caracterizó por importantes tratados y declaraciones en el ámbito territorial, marítimo y político. Comenzando por el ámbito territorial, el Tratado de Lima 
y su Protocolo Complementario, firmado el 3 de junio de 1929, ratificaron los límites entre ambas naciones dejando Arica en Chile y Tacna para el Perú. El Tratado de 1929 puso fin, momentáneamente, a los asuntos pendientes que tenían ambos países desde el fin a la Guerra del Pacífico (1879-1883). El artículo primero indica:

"Queda definitivamente resuelta la controversia originada por el Artículo Tercero del Tratado de Paz y Amistad del veinte de octubre de mil ochocientos ochenta y tres, que era la única dificultad pendiente entre los Gobiernos signatarios" (Dirección de Frontera y Límites, 2014).

El Tratado de 1929 tiene la peculiaridad de haber sido firmado por Augusto Leguía, quien gobernó en el Perú entre los años 1919 y 1930, y que durante su gestión dio solución a los complejos problemas limítrofes que tenía con sus vecinos. Vale destacar que Leguía siempre fue un duro en las negociaciones con Chile, por lo que, la solución adoptada en su gobierno fue más bien pragmática, antes que representativa de la idea política que se manejaba en relación a lo justo (Bákula, 2006).

Otro evento significativo en la relación bilateral, y una primera demostración de buena vecindad, fue la participación de Chile junto a Brasil, Estados Unidos y Argentina como garantes del protocolo de Río de Janeiro de 1942, que ponía fin a la guerra acontecida un año antes entre el Perú y Ecuador.

Entre las décadas de 1940 y 1950, el ámbito marítimo fue el tema principal de la agenda bilateral. En ellas se levantó una inédita propuesta entre Chile y Perú para evaluar y extender la proyección marítima vigente, con el fin de crear las condiciones necesarias de subsistencia, fortalecer el desarrollo económico y cuidar la conservación y protección de los recursos naturales. Dicha iniciativa conjunta generó cambios en el Derecho Internacional del Mar. En el año 1947 se realizaron declaraciones jurisdiccionales unilaterales, entre los dos Estados, con el fin de cambiar las 3 millas marítimas territoriales a 200 millas. El 18 de agosto del año 1952 ambos países, junto a Ecuador, celebran y ratifican la "Declaración sobre Zona Marítima” o "Declaración de Santiago" donde:

“proclaman como norma de su política internacional marítima, la soberanía y jurisdicción exclusivas que a cada uno de ellos corresponde sobre el mar que baña las costas de sus respectivos países, hasta una distancia mínima de 200 millas marinas desde las referidas costas" (Chile. Dirección de Fronteras y Límites b, 2014). 
Este nuevo hito en soberanía quedaría incluido en el Convenio de Derecho del Mar del año 1982. Manuel Rodríguez Cuadros, relata el hecho en su libro "Delimitación marítima con equidad. El caso de Perú y Chile" de la siguiente forma:

“El verdadero impulso que inició el proceso de cambio del Derecho Internacional del mar provino de las iniciativas de Chile y del Perú en 1947. Ambos países aprobaron sendos decretos que, por primera vez en la historia, reivindicaron la soberanía y jurisdicción exclusivas sobre las columnas de aguas oceánicas hasta una distancia de 200 millas, incluidos el suelo, el subsuelo, con la finalidad de preservar, proteger y explotar sus recursos marinos renovables y no renovables" (Rodríguez Cuadros, 2007: 167-168).

Como continuación del "Convenio sobre Zona Especial Fronteriza Marítima” de 1954 se realizó el "Acta de los Representantes de Chile y del Perú" de 1968 y el "Acta de la Comisión Mixta Chileno-Peruana" de 1969 (Chile. Dirección de Fronteras y Límites c, 2014). Esta fue una década en que la continuidad de integración se encaminó tras la creación de la "Corporación Andina de Fomento" actualmente la "Comunidad Andina de Naciones". El ex Canciller de Chile, Valdés Subercaseaux, señala ese episodio en sus memorias, describiendo su experiencia como Canciller tras una reunión de la ALALC, en Uruguay, en la que vio que el camino no era promisorio y de vuelta del viaje junto con sus pares del Perú, Ecuador, Colombia y Venezuela, se les ocurrió crear la CAF:

"El pacto fue firmado en Lima y se agregó el gobierno de Bolivia, cuyo ministro de Relaciones -un militar- nos retrasó la firma varias horas, porque él no quería que antes nos prometiéramos a darle a su país una salida soberana al mar. Después de una larga discusión, yo mantuve mi negativa y la frase que el delegado boliviano deseaba incluir fue puesta al final, después de nuestras firmas, como una condición que sólo obligaba a Bolivia" (Valdés, 2009: 157).

Desde que se firmó el Tratado de 1929, Chile con el Perú lograron construir una dinámica relación que partió con la aclaración de los asuntos territoriales y luego marítimos, que la visión realista de las relaciones internacionales considera vitales para controlar el conflicto. Como complemento, se creó una institución de cooperación económica, cualidad importante para lograr una integración bajo la óptica liberal.

Tomando lo expuesto al inicio del artículo, este primer período pudo ser considerado como otro punto de inflexión en la relación bilateral, puesto que se estaban respetando los tratados, se consolidaban nuevos y se actuaba de forma conjunta por un bien mayor 
como fue el Protocolo de Río de 1942 y la Declaración de Santiago por la delimitación marítima. Pero, como se advertirá a continuación, con la instauración de los regímenes militares se rompen las relaciones bilaterales y se retrocede a un estado de crisis.

En el segundo período, que comienza en la década del 60, se produce la convergencia en Presidentes ideológicamente símiles, Eduardo Frei Montalva (1967-1970) y Fernando Belaúnde (1963-1968), y luego entre el "Gobierno Revolucionario de las Fuerzas Armadas" de Velasco Alvarado (1968-1975) y Salvador Allende (1970-1973), pero que se rompe tras el inicio del Régimen Militar del General Augusto Pinochet (1973-1990), que le tocó estar con Velasco Alvarado; Francisco Morales Bermúdez (1975-1980), Belaunde Terry (1980-1985) y Alan García (1985-1990).

Especialmente, entre los años 1973 y 1978, surgió un fuerte ambiente de desconfianza y alejamiento, debido en gran parte a que se acercaba el centenario de la Guerra del Pacífico. En 1974 hubo una tensión evidente, puesto que ambas fuerzas armadas estaban movilizadas y desplegadas en la frontera (Robles, 2006). El cuadro militar estratégico estaba totalmente hecho para una acción bélica. La revista ¿Qué Pasa? en su edición del 3 de julio de 1993, en un reportaje afirma:

“Con préstamos soviéticos a largo plazo y de baja tasa de interés, Perú inició sus órdenes de compra en Moscú adquiriendo 200 tanques T-54 y T-55, aviones de combate sofisticados, artillería pesada, etc. se irían acumulando año a año en los regimientos peruanos” (Revista ¿Qué Pasa? 1993: 4).

Tal idea también es sostenida por Rodríguez Elizondo (2004) en su libro "El Siglo que vivimos en peligro", en donde señala que el armamentismo peruano no llegó a la guerra por la situación interna del país, pero que en 1975 pudo haber habido guerra, o inclusive, años después al celebrarse el centenario de la Guerra del Pacífico en 1979.

En el invierno de 1975, va a disminuir la tensión de la relación bilateral, luego que el general Francisco Morales Bermúdez tomara el poder del gobierno peruano, ante un General Velasco muy deteriorado por motivos de una enfermedad. Dichos tiempos de serenidad se quebraron en 1978 cuando en diciembre:

"Dos oficiales navales chilenos del petrolero Beagle, ubicado en Talara, fueron detenidos bajo la acusación de haber sido sorprendidos fotografiando instalaciones militares. El 20 de diciembre, la Cancillería peruana informó sobre la expulsión de seis chilenos, incluyendo cuatro funcionarios de rango diplomático. El 19 de enero de 
1979, se declaró persona non grata al embajador de Chile en Lima y se solicitó su retiro inmediato (...) el 20 de enero de 1979 es fusilado un suboficial de la Fuerza Aérea del Perú acusado de haber entregado información militar a los chilenos" (Gallardo, 2005: 216-217).

La tensión político militar no decreció hasta el retorno de la democracia con los presidentes Aylwin en Chile (1990-1994) y Fujimori en Perú (1990-2000). El tercer período entre los Estados comienza con el retorno a la democracia, donde se retoman las relaciones y vuelve a la agenda bilateral el Tratado de 1929, puesto que se quiere concluir con los asuntos que aún no se ejecutaban en su totalidad. Luego de negociaciones bilaterales entre las Cancillerías, en noviembre del año 1999, se firma el "Acta de Ejecución" y se declaran por terminados los temas pendientes entre ambos Estados. El Ministro peruano de Relaciones Exteriores de la época, Fernando de Trazeginies, indicó en su discurso posterior a la firma que:

"Hemos presenciado hace unos minutos, el fin a las últimas secuelas de un conflicto entre Perú y Chile que tuvo lugar hace 120 años y que, felizmente, se encuentra hoy totalmente superado. Desde esa época hasta nuestros días, ha tenido lugar un proceso de curación de las heridas ocasionadas por la guerra y de restablecimiento de las relaciones normales entre nuestros países" (Sandoval, 2011).

De este modo se concluyó de manera definitiva con los asuntos pendientes del mencionado Tratado y su Protocolo Complementario, preservando el Perú sus derechos de usufructo y servidumbre de un muelle, de un malecón de atraque, establecimientos y zonas de operación dentro del Complejo Portuario de la ciudad fronteriza de Arica; así como el libre tránsito de personas, mercaderías y armas entre Tacna y el Muelle de Arica y viceversa (Sandoval, 2011). Luego de que se firmó el Acta de Ejecución y su respectivo Reglamento, el 7 de marzo 2000, se inauguró el Cristo de la Concordia sobre el Morro de Arica, hito que simbolizaba el fin de los asuntos pendientes y daba paso a un nuevo escenario de las relaciones entre ambos Estados. A su vez, el entonces Ministro de Relaciones Exteriores de Chile, Juan Gabriel Valdés, declaró:

"Mediante este acto, completamos la ejecución de algunas cláusulas del Tratado de 1929 y de su Protocolo Complementario, respectivamente los Artículos $5^{\circ}$ y $2^{\circ}$. Después de 70 años, hemos encontrado la forma de terminar una tarea en esta materia, y ofrecer a nuestras comunidades nacionales, una perspectiva que permita mirar hacia el futuro con renovada confianza y en un marco de sólida cooperación” (Sandoval, 2011). 
Concluye el siglo XX con un promisorio horizonte para la relación bilateral. Se acordó la creación del Comité de Seguridad y Defensa (COSEDE), la instauración de mecanismos de intercambio y coordinación entre Estados Mayores de la Defensa Nacional y de las Fuerzas Armadas. Estas instancias, implementadas el año 2001, contaron con versiones posteriores, pero de manera discontinua hasta el día de hoy.

Este tercer período fue idóneo para lograr un punto de inflexión, considerando la ejecución completa del acta complementaria del Tratado de 1929 y los avances en las relaciones bilaterales al punto de crear un proyecto conjunto en materias de seguridad y defensa, pero el debate por límite marítimo volvería a desechar la posibilidad de poner fin a la conformación territorial de los Estados.

\section{Conclusiones}

El fallo de la Corte Internacional de Justicia no cambió la relación bilateral entre Chile y el Perú. La propuesta de punto de inflexión se desmanteló, quedó en el plano especulativo, a causa del denominado "triángulo terrestre". Vale destacar que la propuesta de punto de inflexión fue afirmada públicamente por los Presidentes de ambas Repúblicas, no fue únicamente una idea del mundo público o académico, demostrando que no valieron las buenas intenciones declarativas de los entonces Presidentes Piñera y Humala principalmente.

Retomando la pregunta de investigación ¿Qué explica que el triángulo terrestre no permitiera la posibilidad de punto de inflexión histórico en la relación bilateral? Se responde positivamente con la hipótesis presentada: La propuesta del triángulo terrestre como punto de inflexión que concluiría el ciclo de disputas a lo largo de la historia de la relación bilateral respecto a la conformación territorial, no fue viable de acuerdo a la incidencia del pasado en la situación actual. Esta afirmación se basa en dos elementos expuestos en el artículo y que se resumen a continuación.

Primero, frente al contexto de la búsqueda de un punto inflexión, el artículo expone dos momentos en que pudo haber existido y no se produjo: tras la firma del Tratado de 1929 y tras el Acta de Ejecución a fines de los años 90. Ambos momentos tienen como elemento común, que se desprenden de la Guerra del Pacífico y de los tratados que los secundaron. En consecuencia, que tras el fallo de la CIJ no se haya producido el punto de inflexión 
histórico, no es una novedad en la historia bilateral, al contrario es un intento fallido adicional en la búsqueda de finiquitar los asuntos pendientes de aspecto territorial, lo que deja manifiesto la inconstancia en la integración bilateral.

La hipótesis de punto de inflexión fue rechazada dado que la conformación territorial de los Estados volvió a ser incompatible y que en el corto y mediano plazo no se le ve solución. Esto se explica por la carga histórica que tienen ambos países, dada por el origen de la relación, muy marcada por la herencia negativa de la Colonia, la Guerra del Pacífico con sus consecuentes tratados y la falta de integración continua durante el Siglo XX. Cabe mencionar que respecto a la Guerra del Pacifico, ésta trajo beneficios territoriales a Chile en desmedro del Perú; marcó una parte de la identidad nacional del Perú en oposición a Chile; y forjó una identidad política bilateral que no ha sido modificada hasta el día de hoy y que tiene como cualidad la crisis y la confrontación diplomática por la conformación territorial.

Segundo, el denominado triángulo terrestre, que es de 3,7 hectáreas, vale más que un cambio en la relación bilateral para ambos Estados, lo cual tiene una relación directa con la conformación de ambos Estados y sus territorios. Como señala Robledo en la cita tomada en este artículo, el punto de inflexión dependía de la aceptación de la conformación territorial entre ambas partes del fallo de la Corte, asunto que no ocurrió. El denominado "triángulo terrestre" no tiene un valor cuantitativo al día de hoy, pero su cualidad histórica y simbólica es muy relevante. En razón de ello ninguno de los Estados está dispuesto a priorizar unas buenas relaciones bilaterales por sobre la oportunidad de reclamar un espacio territorial como propio.

Según la discusión conceptual realizada en la primera sección de este artículo, un punto de inflexión es aquel evento que demarca un cambio significativo que circunscribe un antes y después. En este caso, se traduce como un antes y un después en la historia de la relación bilateral entre Chile y el Perú que ha estado demarcada por situaciones de crisis y conflicto más que de cooperación. Contrariamente, entre ambos Estados existen ciclos de integración y conflicto diplomático, no una relación lineal que se consolide en el tiempo. 


\section{Bibliografía}

Bákula, J. (2006). El Perú en el Reino Ajeno. Historia interna de la acción externa. Lima: Universidad de Lima y Fondo Editorial.

Bolivia. Dirección Estrategia de Reivindicación Marítima-DIREMAR. (2014). “Tratado de 1866" (en línea). (Fecha de consulta 10 de diciembre de 2014). Disponible en: http:// www.diremar.gob.bo/node/81

Cambridge Dictionary. (2014). "Turning points" (en línea). (Fecha de consulta 10 de diciembre de 2014) Disponible en: http://dictionary.cambridge.org/dictionary/english/ turning-point /

Cavieres, F. (2006). Chile-Perú, La Historia y La Escuela. Conflictos Nacionales, Percepciones Sociales. Valparaíso: Ediciones Universitarias de Valparaíso.

Cayo, P. (2010). "Perú y Bolivia: una historia común” Lima: Editorial de la Universidad del Pacifico.

Chile. Biblioteca del Congreso Nacional. (1823). "Acuerdo de auxilio al Perú 1823" (en línea). (Fecha de consulta 22 de septiembre de 2014) Disponible en: https://www.leychile. $\mathrm{cl} /$ Consulta/listaresultadosimple? cadena=Acuerdo+de+auxilio + al+Perú +1823 .

Chile. Dirección de Fronteras y Límites. (2014). “Tratado de 1929” (en línea). (Fecha de consulta 22 de septiembre de 2014) Disponible en: http://www.difrol.gob.cl/instrumentosjuridicos/peru.html.

Chile. Dirección de Fronteras y Límites. (2014b). "Declaración sobre Zona Marítima, 18 de agosto de 1952" (en línea). (Fecha de consulta 30 de septiembre de 2014) Disponible en: http://www.difrol.gob.cl/instrumentos-juridicos/peru.html.

Chile. Dirección de Fronteras y Límites. (2014c). "Acta de los Representantes de Chile y del Perú, 26 de abril de 1968” (en línea). (Fecha de consulta 30 de septiembre de 2014) Disponible en: http://www.difrol.gob.cl/instrumentos-juridicos/peru.html.

Corte Internacional de Justicia. (2008). "Peru institutes proceedings against Chile with regard to a dispute concerning maritime delimitation between the two States", en 
International Court of Justice. Cases. List of all cases (en línea). (Fecha de consulta 10 de octubre de 2014). Disponible en: http://www.icj-cij.org/docket/index.php?p1=3\&p2=3\& code $=$ pch \&case $=137 \& \mathrm{k}=88 \& \mathrm{p} 3=0$.

Corte Internacional de Justicia. (2014). "The Court determines the course of the single maritime boundary between Peru and Chile", en International Court of Justice. Press Relase, 2014 (en línea). (Fecha de consulta 10 de octubre de 2014). Disponible en: http:// www.icj-cij.org/docket/index.php?p1=3\&p2=3\&k=88\&case $=137 \& \operatorname{code}=$ pch $\& p 3=5$.

El Mercurio. (2012) "Presidentes de Chile y Perú se comprometen a respetar fallo de La Haya” en Diario EMOL, 30 de noviembre de 2012, (en línea). (Fecha de consulta 10 de octubre de 2014). Disponible en: www.emol.com.

El Mercurio. (2014). "Ollanta Humala asegura que el "triángulo terrestre" pertenece a Perú", en Diario EMOL, 10 de febrero de 2014, (en línea). (Fecha de consulta 10 de octubre de 2014) Disponible en: www.emol.com.

ICSO-UDP. (2013). "Charla en la Universidad Diego Portales, de los ex Cancilleres de Chile, Gabriel Valdés y del Perú, Rafael Roncagliolo". Santiago de Chile, 25 de Septiembre de 2013.

Gallardo, A. (2005). Crisis Internacionales en Sudamérica: Teoría y Análisis. Santiago de Chile: Ediciones de la Academia Nacional de Estudios Políticos y Estratégico- ANEPE.

Launius, R. (2007). What Are Turning Points in History, and What Were They for the Space Age. En: Launius, R. y Dick, J. (editores) Societal Impact of Spaceflight (pp. 19-40). Washington D.C.: NASA.

Launius, R. (2007). What Are Turning Points in History, and What Were They for the Space Age. En: Societal Impact of Spaceflight, Washington D.C.

La Tercera. (2014). "Canciller responde a Ministro del Interior de Perú: triangulo terrestre es incuestionablemente chileno" en Diario La Tercera, 28 de agosto de 2014, (en línea). (Fecha de consulta 10 de octubre de 2014). Disponible en: www.latercera.cl.

Merriam Dictionary. (2014). “Turning Point” (en línea). (Fecha de consulta 10 de octubre de 2014) Disponible en: http://www.m-w.com/dictionary/turning\%20point. 
Milet, P. (2004). Chile-Perú: Las dos caras de un espejo. Revista de Ciencia Política 24(2), 228-235.

Milet, P. (2005). Chile-Perú: las raíces de una difícil relación. Revista de Estudios Internacionales 38(150), 59-73.

Perú. Ejército. (2014). "Significado del Escudo del Ejército del Perú” (en línea). (Fecha de consulta 22 de septiembre de 2014). Disponible en: www.ejercito.mil.pe/index.php/ nuestra-heraldica.

Perú. Ministerio de Relaciones Exteriores. (2010). "Relaciones bilaterales con Chile" (en línea). (Fecha de consulta 22 de septiembre de 2014). Disponible en: http://www.rree.gob. pe/SitePages/america_sur.aspx\#chile.

Perú, Ministerio de Relaciones Exteriores del Perú. (2013). "Declaración de los Ministro de Relaciones Exteriores de Chile y el Perú 24 de enero de 2013" en Declaraciones conjuntas (en línea). (Fecha de consulta 10 de octubre de 2014). Disponible en: http://www.rree. gob.pe/SitePages/comunicados.aspx\#conjuntos.

Perú, Presidencia. (2014). "Presidente Humala presenta Carta del Límite del a frontera sur” (en línea). (Fecha de consulta 10 de octubre de 2014) Disponible en: http://www. presidencia.gob.pe/lista-notas-prensa.

Publimetro Perú. (2014). "Daniel Parodi: tenemos mucho en común. En Publimetro Perú" 27 de enero de 2014 (en línea). (Fecha de consulta 10 de octubre de 2014). Disponible en: http://publimetro.pe/actualidad/noticia-daniel-parodi-tenemos-mucho-comun-19926.

Real Academia Española. (2014). "Inflexión” (en línea). (Fecha de consulta 8 de octubre de 2014) Disponible en: http://dle.rae.es/srv/search?m=30\&w=inflexión.

Revista ¿Qué Pasa? (1993). "Los años que remecieron Chile” Edición del 3 de julio de 1993, 1-14.

Robles, M. J. (2006). De la disuación a la cooperación: dos siglos en la relación PerúChile. Revista Fuerzas Armadas y Sociedad 20(1), 31-50.

Robledo, M. (2012). Chile-Perú ¿punto de inflexión? Revista Mensaje 608, 29-31. 
Rodríguez Cuadros, M. (2007). Delimitación marítima con equidad, el caso de Perú y Chile. Lima: Peisa.

Rodríguez Elizondo, J. (2004). Chile-Perú, el siglo que vivimos en peligro. Santiago de Chile: La Tercera Mondadori.

Rodríguez Elizondo, J. (2009). De Charaña a La Haya. Chile entre la aspiración marítima de Bolivia y la demanda marítima de Perú. Santiago de Chile: La Tercera Mondadori.

Sandoval, A. (2011). "Perú-Chile. Instrumentos jurídicos internacionales para el establecimiento de su frontera (1883-1999)", (en línea). (Fecha de consulta 11 de noviembre de 2011). Disponible en: http://www4.congreso.gob.pe/comisiones/1999/exteriores/ chile/INDICE.html.

Valdés, G. (2009). Gabriel Valdés, sueños y memorias. Santiago de Chile: Editorial Taurus.

Yepes, E. (1999). Un plebiscito imposible. Tacna y Arica 1925-1926. Lima: Ediciones Análisis.

Winter, L. (2013). La defensa de Chile en La Haya. Santiago de Chile: Ediciones LYD.

Zapata, A. (2012). "Clases Diplomado "Chile-Perú: puntos de encuentro" realizado por la Fundación Konrad Adenauer y el Instituto de Estudios Internacionales de la Universidad de Chile. 


\section{Anexo I: Mapas del Fallo de la Corte Internacional de Justicia, en el caso del Perú contra Chile, en relación de la zona en controversia y de la resolución de la Corte}

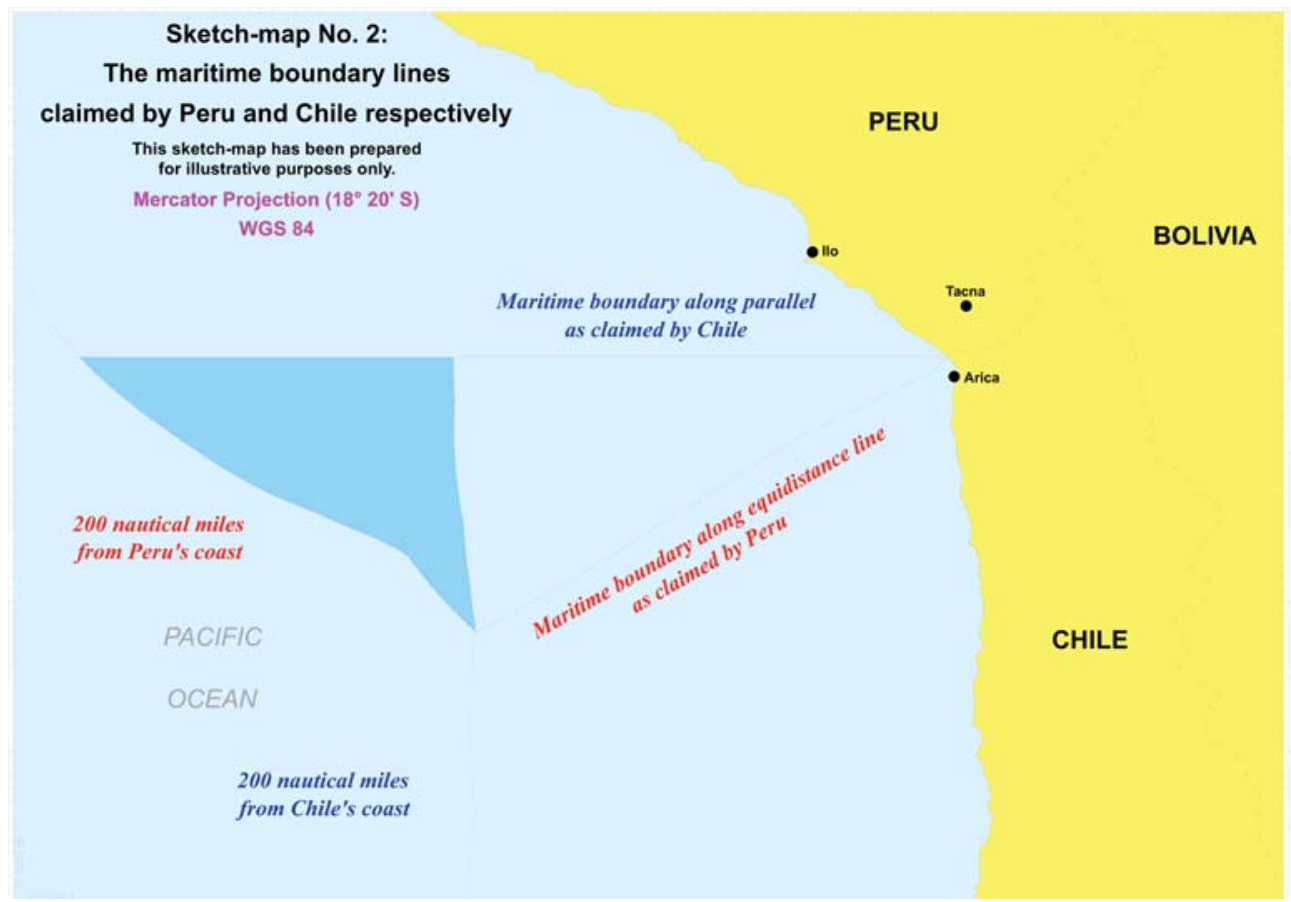




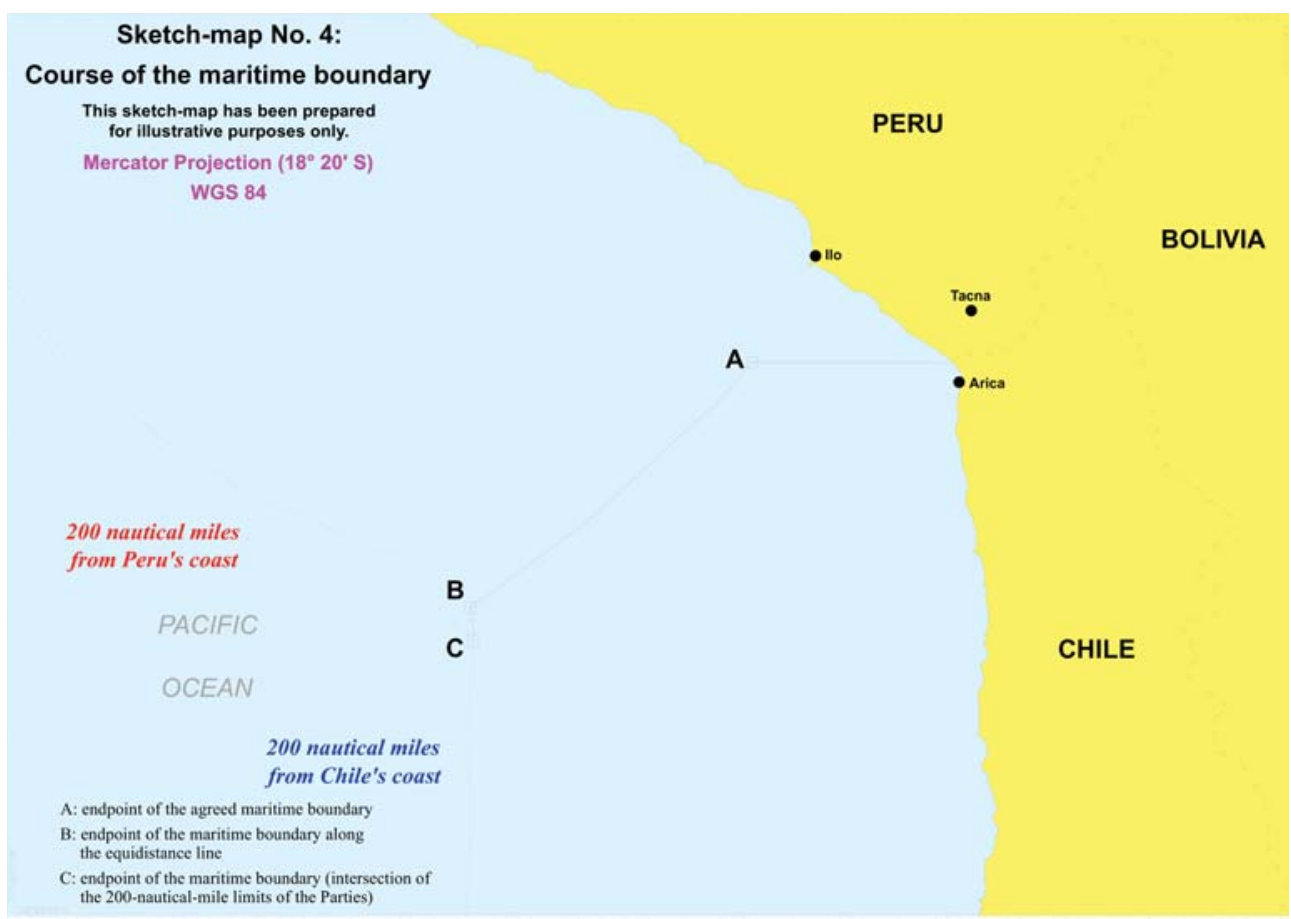

Corte International de Justicia. (2014) Perú against Chile, Press release. (Fecha de consulta 11 de noviembre de 2011). (en línea) Disponible en: http://www.icj-cij.org/ docket/files/137/17928.pdf 


\section{Anexo II: Mapa alegórico del denominado triángulo terrestre}

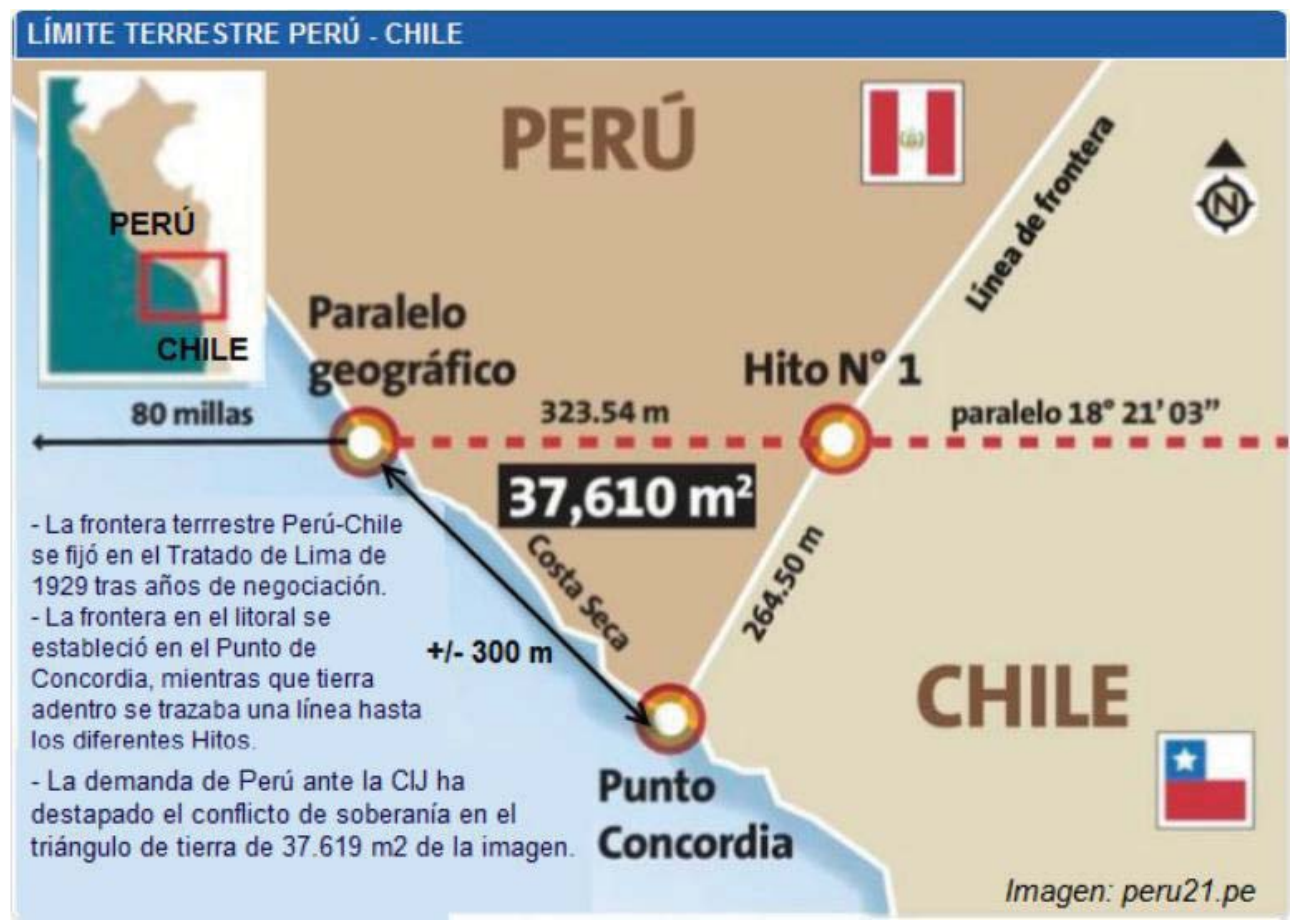

LatinoAmericaHoy. (2014). La frontera marítima Perú-Chile y el triángulo terrestre. (Fecha de consulta 11 de noviembre de 2011). (en línea) Disponible de: https:// latinamericahoy.es/2014/09/04/la-controversia-en-la-frontera-maritima-peru-chile-yel-triangulo-terrestre/ 